\section{Isolation, Characterization, and Expression Analysis of the MaMDH Gene in Banana}

\author{
Cai-Hong Jia ${ }^{1}$ and Ju-Hua Liu ${ }^{1}$ \\ Institute of Tropical Bioscience and Biotechnology, Chinese Academy of \\ Tropical Agricultural Sciences, Xueyuan Road 4, Longhua County, Hainan \\ 571101, China
}

\section{Zhi-Qiang Jin}

Institute of Tropical Bioscience and Biotechnology, Chinese Academy of Tropical Agricultural Sciences, Xueyuan Road 4, Longhua County, Hainan 571101, China; and Haikou Experimental Station, Chinese Academy of Tropical Agricultural Sciences, Hainan 570101, China

\section{Qiu-Ju Deng, Jian-Bin Zhang, and Bi-Yu Xü ${ }^{2}$}

Institute of Tropical Bioscience and Biotechnology, Chinese Academy of Tropical Agricultural Sciences, Xueyuan Road 4, Longhua County, Hainan 571101, China

Additional index words. banana (Musa acuminata L. AAA group), MDH, isolation, expression patterns

\begin{abstract}
A full-length cDNA isolated from banana (Musa acuminata L. AAA group) fruit was named $M a M D H$, containing an open reading frame encoding 332 amino acids that represents the gene for cytoplasmic malic dehydrogenase (MDH). Sequence analysis showed that $M a M D H$ shares high similarity with $M D H s$ from castor bean (XP_002533463), tobacco (CAC12826), peach (AAL11502), and chickpeas (CAC10208). Real-time quantitative polymerase chain reaction (PCR) analysis of $M a M D H$ spatial expression showed that it was expressed in all organs examined: roots, rhizomes, leaves, flowers, and fruits. The expression was the highest in flowers followed by the fruits and roots, whereas the rhizomes and leaves displayed the lowest expression levels. Real-time quantitative PCR revealed that $M a M D H$ exhibited differential expression patterns in post-harvest banana fruits correlating with ethylene biosynthesis. In naturally ripened banana fruits, $M a M D H$ expression was in accordance with ethylene biosynthesis. In accordance, for banana fruits treated with the ethylene analog 1-methylclopropene (1-MCP), MaMDH expression levels were inhibited and remained constant. After treatment with ethylene, $\mathrm{MaMDH}$ expression in banana fruits significantly increased with ethylene biosynthesis and peaked 3 days after harvest, which was 11 days earlier than that in naturally ripened banana fruits. These results suggest that $M a M D H$ expression is induced by ethylene to regulate post-harvest banana fruits ripening.
\end{abstract}

Malic acid dehydrogenase (MDH) exists widely in living organisms and is a key enzyme in biological sugar metabolism that catalyzes the oxidation of malic acid to oxaloacetic acid in a reversible fashion. According to the coenzyme specificity, MDH can be divided into two subgroups that are dependent on either NAD (NAD-MDHs) or NADP (NADP-MDHs). MDH, an important metabolic

Received for publication 21 Mar. 2013. Accepted for publication 22 Oct. 2012.

This work was supported by the Ministry of Science and Technology of the People's Republic of China (No. 2011AA10020605), the Modern Agroindustry Technology Research System (CARS-32), the Central Level, and Scientific Research Institutes for Basic R \& D Special Fund Business (1630052012003)

${ }^{1}$ These authors contributed equally to this work.

${ }^{2}$ To whom reprint requests should be addressed; e-mail biyuxu@126.com. stages (Etienne et al., 2002). Likewise, the expression of the cyMDH in apples gradually increased during fruit development and maturation, resulting in enhanced cyMDH activity simultaneously (Yao et al., 2011b). Both malate and fructose content increased the effect of elevated $c y M D H$ expression in apple calluses and tomatoes. In wheat, cyMDH mainly catalyzed synthesis of malic acid produced in response to stress conditions (Ding and $\mathrm{Ma}$, 2004). Other studies reported that $c y M D H$ played a role in enhancing plant resistance to aluminum, salt, and chilling injury as well as facilitating seed germination, cell growth, and pollen development (Wang et al., 2010; Yao et al., 2011a).

Banana (Musa acuminata L. AAA group cv. Brazilian) is a typical climacteric fruit, which is largely cultivated in tropical and subtropical regions. The pattern of ethylene production during banana fruit ripening differs from other climacteric fruits with a sharp rise and fall in the rate of ethylene production occurring during the early climacteric rise in respiration (Liu et al., 1999). There are many physiological and biochemical changes in the post-harvest maturation process such as polyphenol and chlorophyll degradation, aromatic substance formation, and changes in carbohydrate levels. These changes all directly affect the quality, flavor, color, and postharvest shelf life of banana fruits (Giovannoni, 2004). Therefore, research on the development of banana fruits and the regulation of postharvest maturation is important for improving banana fruit quality and prolonging shelf life. At present, there is little research concerning basal metabolic enzymes in banana, and there are no reports on the $M D H$ gene in banana. In this study, we cloned the cytoplasmic malic acid dehydrogenase gene $M a M D H$ from banana fruits using cDNA probes with the quick end amplification method [rapid amplification of cDNA ends (RACE)] and analyzed the sequence using BLAST $(<\mathrm{http}: / /$ ncbi.nlm.nih.gov/blast $>)$ and SOPMA $(<\mathrm{http}: / /$ npsa-pbil.ibcp.fr/cgi-bin/>). We also used fluorescent quantitative PCR to evaluate $M a M D H$ expression in different organs of banana plants and post-harvest fruits exposed to different treatments. The result of this study provides a certain basis for further research on enzyme metabolism of banana fruits and contributes to understanding the molecular mechanism of banana and climacteric fruit during the maturation process.

Kochianl et al , 2004, Ma et al, 2001, Yao et al., 2011). As a result of its multifaceted activities, there are many MDH isozymes in plant cells. Depending on coenzyme specificity, cellular localization, and physiological function, these isozymes can be divided into five categories: chloroplast NAD-MDH, chloroplast NADP-MDH, mitochondria NAD-MDH $(\mathrm{mmDH})$, microbody NAD-MDH (including glyoxysomal $\mathrm{MDH}$ and peroxisomal $\mathrm{MDH}$ ), and cytoplasmic NAD-MDH (cyMDH) (Gietl, 1992; Ocheretina and Scheibe, 1997). At present there is a lot of research on cyMDH, but its function is not understood. In peaches, expression of cyMDH in ripe fruits was higher than that in fruits in the early developmental

\section{Materials and Methods}

Banana fruits and treatments. Banana (M. acuminata L. AAA group, cv. Brazilian) fruits were provided by the banana plantation of the Institute of Tropical Bioscience and Biotechnology (Chengmai, Hainan) and were harvested at the mature green stage (100 to $110 \mathrm{~d}$ after flower shooting). Banana fruit hands of similar developmental stages were selected and three fingers from the hands were divided into three groups for different treatments. For natural ripening, the group of banana fruits was kept at $25^{\circ} \mathrm{C}$ and allowed 
to ripen naturally. For ethylene treatment, the group of banana fruits was treated with $100 \mu \mathrm{L} \cdot \mathrm{L}^{-1}$ ethylene in an airtight container for $24 \mathrm{~h}$ at $25^{\circ} \mathrm{C}$ to initiate ripening. For ethylene inhibition treatment, 1-MCP was used to interfere with ethylene responses; banana fruits were sealed in an airtight container and treated with $1 \mu \mathrm{L} \cdot \mathrm{L}^{-1} 1-\mathrm{MCP}$ for $24 \mathrm{~h}$ at $25^{\circ} \mathrm{C}$. The treated fruits were allowed to ripen at $25{ }^{\circ} \mathrm{C}$ and samples were then frozen in liquid $\mathrm{N}_{2}$ and stored at $-80{ }^{\circ} \mathrm{C}$ for subsequent extraction of total RNA at different ripening stages. The experiments were repeated at least three times.

Measurement of ethylene production. Ethylene evolution was measured by enclosing fruit samples in an airtight container for $2 \mathrm{~h}$ at $25{ }^{\circ} \mathrm{C}$, withdrawing $1 \mathrm{~mL}$ of the headspace gas, and injecting the gas into a gas chromatograph (GC-2010; Shimadzu, Kyoto, Japan) fitted with a flame ionization detector and an activated alumina column. Ethylene production measurements were obtained as recommendations by the manufacturer. The experiments were repeated at least three times.

RNA extraction and $c D N A$ synthesis. For the expression at the tissue-specific level, total RNA was separately extracted from the roots, rhizomes, leaves, flowers, and fruits of 8-month-old plants using the cetyltrimethylammonium bromide (CTAB) method (Asif et al., 2000).

For cloning of full-length cDNA, total RNA from banana fruit tissues (including peel and pulp) was isolated $2 \mathrm{~d}$ after harvest using the CTAB method. First-strand cDNA was synthesized using the SMART ${ }^{\mathrm{TM}}$ PCR cDNA Synthesis Kit and SMARTScribe reverse transcriptase (Clontech, Palo Alto, CA) according to the manufacturer's instructions.

Isolation of full-length MaMDH $c D N A$ using RACE-PCR. Based on the entire $3^{\prime}$ cDNA of $M a M D H$, previously cloned by suppression subtractive hybridization ( $\mathrm{SSH}$ ) (Xu et al., 2007), RACE was used to obtain the $5^{\prime}$ end of the cDNA using double-stranded cDNA as a template. The 5'-RACE was performed using the BD SMARTer RACE cDNA Amplification Kit (Clontech) according to the manufacturer's instructions. The amplified products of the $5^{\prime} \mathrm{cDNA}$ ends were inserted into the pGEM-T Easy vector (Promega, Madison, WI) and sequenced by the Sangon Company (Shanghai, China) using an ABI 3770 DNA sequencer. According to the $5^{\prime}$ - and $3^{\prime}$-cDNA regions, a pair of genespecific primers was designed $\left(5^{\prime}-\mathrm{ATGG}\right.$ CAAAAGATCCGGTGC- $3^{\prime}$ and $5^{\prime}$ - CATG TATTCTTGCCTTGGGAC- $3^{\prime}$ ) to obtain fulllength cDNA. The sequence was analyzed by BLAST (<http://ncbi.nlm.nih.gov/blast $>$ ).

Quantitative real-time RT-PCR analysis of $M a M D H$ expression. For real-time quantitative reverse transcription (RT)-PCR, total RNA was extracted from the roots, rhizomes, leaves, flowers, and fruit tissues (including peel and pulp) from naturally ripened banana fruits at $0,2,6,10,12,14$, and $16 \mathrm{~d}$ after harvest; from ethylene-treated fruits at 0,1 , $2,3,4,5$, and $6 \mathrm{~d}$ after harvest; and from
1-MCP-treated fruits at $0,2,6,10,12,14$, and $16 \mathrm{~d}$ after harvest. Poly(A) ${ }^{+}$-mRNA (200 ng) was converted into cDNA using the SMART PCR cDNA Synthesis Kit (Clontech) in a final volume of $20 \mu \mathrm{L}$, which subsequently served as the template for real-time PCR.

The expression level of $M a M D H$ was determined by real-time RT-PCR analysis using Stratagene Mx3000P (Stratagene, CA) and SYBR Green as a fluorescent dye. The reaction mixture consisted of a $25-\mu \mathrm{L}$ solution containing $12.5 \mu \mathrm{L} 2 \times$ SYBR Green PCR Master Mix, $0.5 \mu \mathrm{L}$ ROX reference dye, and $100 \mathrm{ng}$ reverse-transcribed RNA. The primer sets used and their optimal amounts were as follows: MaMDH5' : 5' -TGCTGGCG AATGGACAATC-3' (10 pmol); MaMDH3': 5' - GGCGGCAACAGAGTCAACC-3' (10 pmol), actin5': 5'-CGAGGCTCAATCAA AGA-3' (10 pmol), actin3': 5' -ACCAGCA AGGTCCAAAC-3' (10 pmol). The thermal cycling conditions were $94{ }^{\circ} \mathrm{C}$ for $3 \mathrm{~min}$ followed by 40 cycles at $94{ }^{\circ} \mathrm{C}$ for $7 \mathrm{~s}, 55^{\circ} \mathrm{C}$ for $15 \mathrm{~s}$, and $72{ }^{\circ} \mathrm{C}$ for $20 \mathrm{~s}$. Reactions were performed in triplicate and the data analyzed using $\mathrm{MxPro}^{\mathrm{TM}}$ QPCR software (Stratagene). Actin was used as a reference sample to which the $M a M D H$ product amounts were compared. Differences in $\mathrm{Ct}$ values between the $\mathrm{MaMDH}$ and actin transcripts were expressed as fold changes relative to actin. The experiments were repeated at least three times.

\section{Results}

Cloning and sequence analysis of MaMDH from banana fruits. A cDNA fragment having high similarity to the $M D H$ gene was previously obtained by SSH (Xu et al., 2007). RACE approaches were then used to isolate full-length $M D H$ cDNA from banana fruits. Sequence analysis revealed that the cDNA was $999 \mathrm{bp}$ in length, having an open reading frame encoding a single peptide of 332 amino acid residues with 48- and 250-bp $5^{\prime}$ and $3^{\prime}$ untramslated regions (UTRs), respectively. This gene was named $M a M D H$. The native molecular weight of the MaMDH protein was estimated to be $44.08 \mathrm{kDa}$ with an isoelectric point of 6.26 (data not shown). Conserved domain analysis showed that MaMDH protein had 15 NADbinding sites, eight malic acid-binding sites, and 16 dimer-binding sites. Meanwhile, MaMDH contained the catalytic group element "IWGNH" and NAD binding motif "TGAAGQI" (Fig. 1), which are both widely and highly conserved in cyMDHs from many species, suggesting that this gene encodes a cytoplasmic malate dehydrogenase.

The deduced amino acid sequence of MaMDH also had high sequence identity with MDHs from other plant species, including castor bean $(92 \%)$, tobacco $(92 \%)$, peach (92\%), and chickpeas (92\%) (Fig. 1). The 15 plant $\mathrm{MDH}$ amino acid sequences in the National Center for Biotechnology Information database were aligned by MEGA, which showed that, compared with other species, MaMDH is closer to cytoplasmic $\mathrm{MDH}$. Once again, these suggested that the
$M a M D H$ gene encodes a cytoplasmic malate dehydrogenase (Fig. 2).

Hydropathy profile and secondary structure of MaMDH. Hydrophilicity analysis found that there was a strong hydrophobic domain at the $\mathrm{N}$-terminal (Fig. 3A), which is another widespread feature of cyMDHs from other species. Protein secondary structure is a periodic conformation that arranges the polypeptide chain in a one-dimensional direction through the formation of hydrogen bonds, and its prediction and analysis aid the understanding of the protein's spatial structure. The MaMDH secondary structure was predicted from the amino acid sequence using Self Optimized Prediction Method from Alignment (SOPMA). The secondary structure of the putative MaMDH protein was composed of $45.2 \% \alpha$-helix, $5.7 \%$ $\beta$-turn, $17.2 \%$ extended strand, and $31.9 \%$ random coil. The C-terminal section of the protein had a greater amount of $\alpha$-helix structure (Fig. 3B).

Temporal and spatial transcript accumulation of MaMDH. To clarify the patterns of $M a M D H$ expression in different organs of the banana plant, real-time quantitative RT-PCR was carried out on cDNA from different organs. MaMDH was expressed at different levels in all organs examined: roots, rhizomes, leaves, flowers, and fruits with abundant $M a M D H$ transcripts in flowers, lower expression levels in the roots, rhizomes, and fruits, and the lowest expression levels in leaves (Fig. 4).

Measurement of ethylene evolution during post-harvest banana fruit ripening. Changes in the rate of ethylene production were examined during post-harvest banana fruit ripening. In naturally ripened banana fruits (Fig. 5A), ethylene production began to increase $8 \mathrm{~d}$ after harvest, peaked sharply at $14 \mathrm{~d}$, and then rapidly decreased. For ethylene-treated banana fruits (Fig. 5B), endogenous ethylene production started $1 \mathrm{~d}$ after harvest and reached a maximum $3 \mathrm{~d}$ after harvest, which is 7 and $11 \mathrm{~d}$, respectively, before that of naturally ripened banana fruits. In addition, the maximal level of ethylene production was higher for ethylenetreated banana fruits compared with naturally ripened banana fruits. However, on treatment with the ethylene inhibitor 1-MCP (Fig. 5C), ethylene production started $14 \mathrm{~d}$ after harvest and but its level remained very low.

Differential expression of $\mathrm{MaMDH}$ during banana fruit ripening. Changes in $M a M D H$ expression during post-harvest ripening were examined using real-time RT-PCR. Changes in $M a M D H$ expression in naturally ripened fruits differed from ethylene-treated fruits and occurred at different stages of ripening (naturally ripened: $14 \mathrm{~d}$, ethylenetreated: 3 d) (Fig. 6A). There were marked changes in MaMDH expression levels in ethylene-treated banana fruits during postharvest ripening with MaMDH expression increasing sharply during the period 0 to $3 \mathrm{~d}$ after harvest (Fig. 6B). For 1-MCP-treated fruits, $M a M D H$ expression was essentially constant 0 to $14 \mathrm{~d}$ after harvest and peaked on Day 16 (Fig. 6C). 


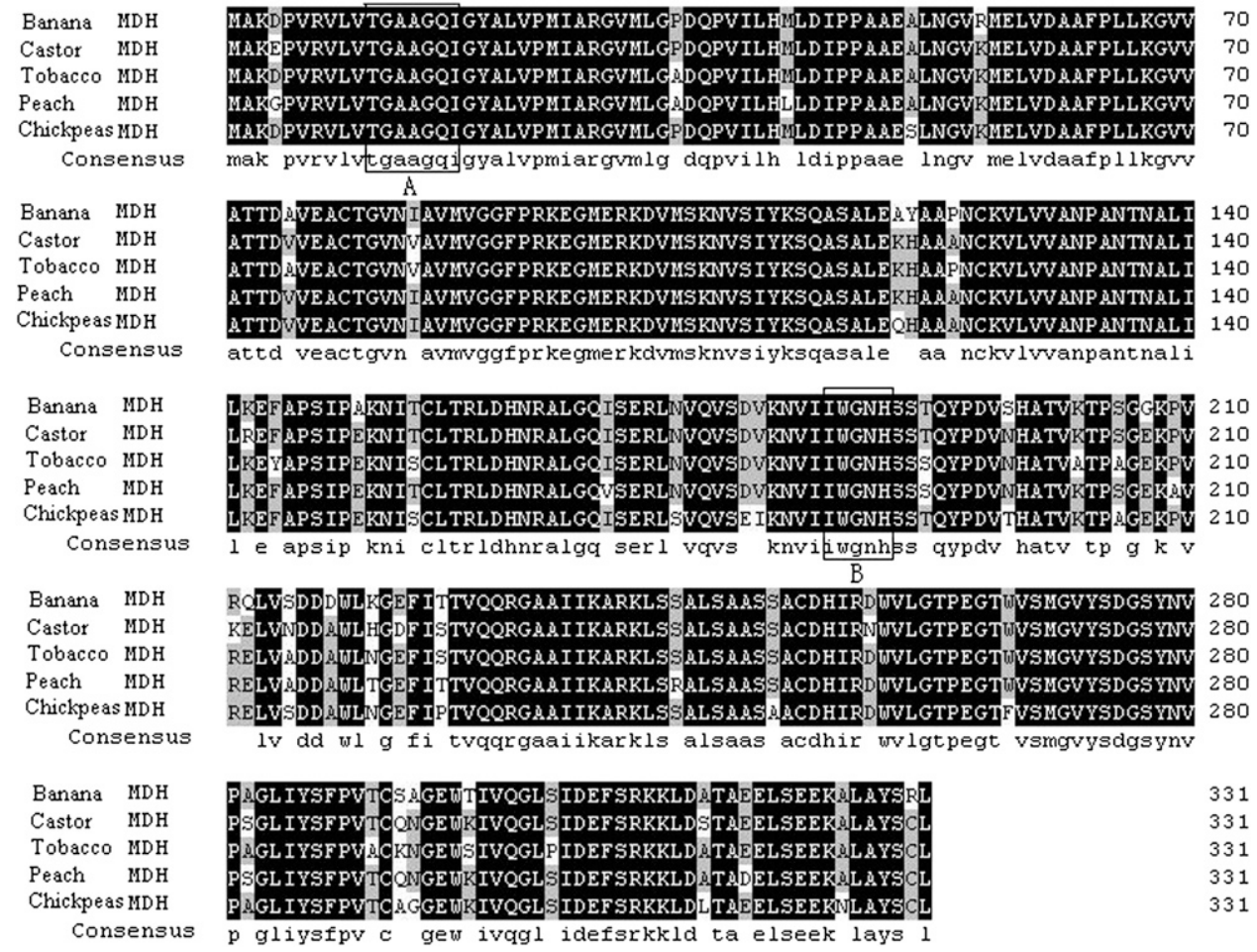

Fig. 1. Alignment of predicted amino acid sequences of MDH genes from different plants. (A) Domain: NAD-combined primitives; (B) domain: catalytic primitives. $\mathrm{MDH}=$ malic dehydrogenase.

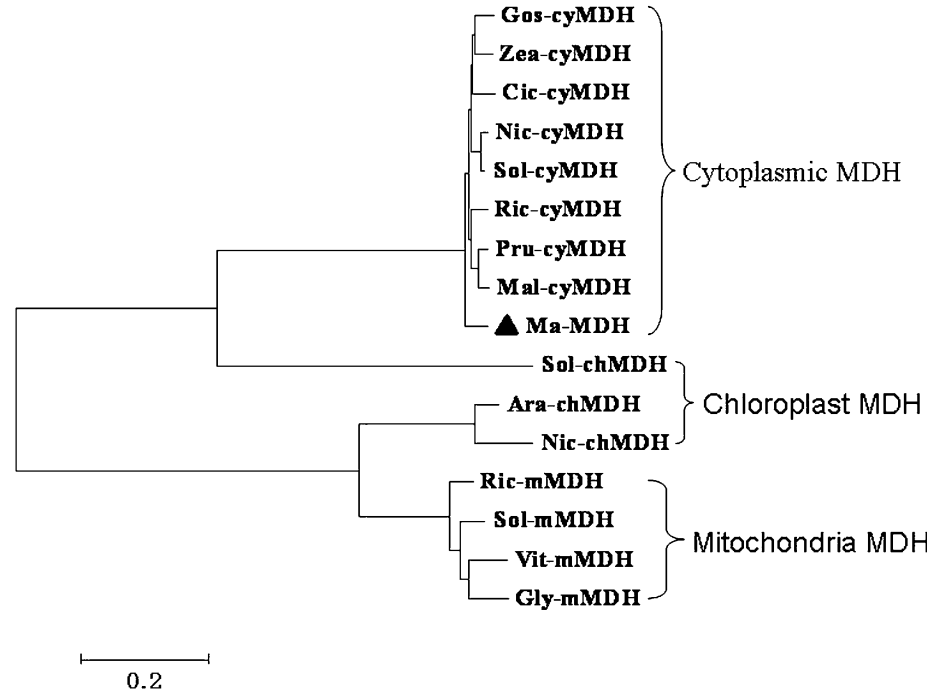

Fig. 2. Phylogenetic tree analysis of the deduced amino acid sequences of malic dehydrogenase (MDH) in different species. The tree was constructed using the MEGA method. cyMDH $=$ cytosolic NADdependent $\mathrm{MDH} ; \mathrm{mMDH}=$ mitochondrial NAD-dependent $\mathrm{MDH}$; chMDH = chloroplast NADPdependent MDH; Pru-cyMDH (AAL11502); Mal-cyMDH (ABB36659); Ric-cyMDH (XP-002533463); Nic-cyMDH (CAC12826); Gos-cyMDH (ACJ11738); Zea-cyMDH (NP-001105603); Cic-cyMDH (CAC10208); Sol-cyMDH (ABC01890); Ara-chMDH (CAA74320); Nic-chMDH (CAB45387); Sol-chMDH (NP001234009); Gly-mmDH (AAD56659); Ric- mмDH (XP-002524262); Vit-mmDH (AF195869); Sol-mmDH (AAU29198). MaMDH (banana).

\section{Discussion}

We cloned the malic acid dehydrogenase gene from the cDNA library of banana fruits and found that $M a M D H$ had a NAD-binding site, malic acid-binding site, and dimer-binding site that are consistent with other members of the cyMDH family. In addition, MaMDH carried the catalytic sequence "IWGNH" and NAD combined site "TGAAGQI," which are two basic elements that are highly conserved in the cyMDHs of many species. These features together indicate that MaMDH may be a member of the cytoplasmic-type malic acid. Homology analysis found that $M a M D H$ encoded a protein that showed high amino acid sequence identity and sequence conservation with several cyMDHs from plants. In terms of cellular localization, MDHs from the same organelles have higher sequence similarity. According to the endosymbiotic theory for the origin of organelles, different MDH family members may have arisen as a result of bacterial invasion of eukaryotic cells (Gray et al., 2001; McAlisterHenn, 1988). However, the relationship between different kinds of MDH is complex, and understanding their evolutionary origins will require future research.

A strong hydrophobic domain 26 amino acids downstream from the first five $\mathrm{N}$ terminal amino acids of MaMDH was found in all cyMDHs from plants, animals, and bacteria. Computer analysis indicated that this domain was unlikely to exist as a presequence or signal peptide. Furthermore, in alfalfa, the N-terminal amino acid sequence of cyMDH protein matched exactly that predicted from the cDNA sequence (Susan et al., 1998). This excludes the possibility of proteolytic of cyMDH peptides. Whether this implies that cyMDHs interact with some membrane system is an interesting hypothesis warranting further investigation.

The secondary structure analysis by molecular modeling MaMDH was mainly constituted with $\alpha$-helix and random coil, which were interlaced with $\beta$-turn and extended strand. The structures of bend, turn, and random coil are essential for the MDH reaction, because both the catalytic site and NAD-binding domain are located in these structural domains. 
A

Protscale output for user sequence

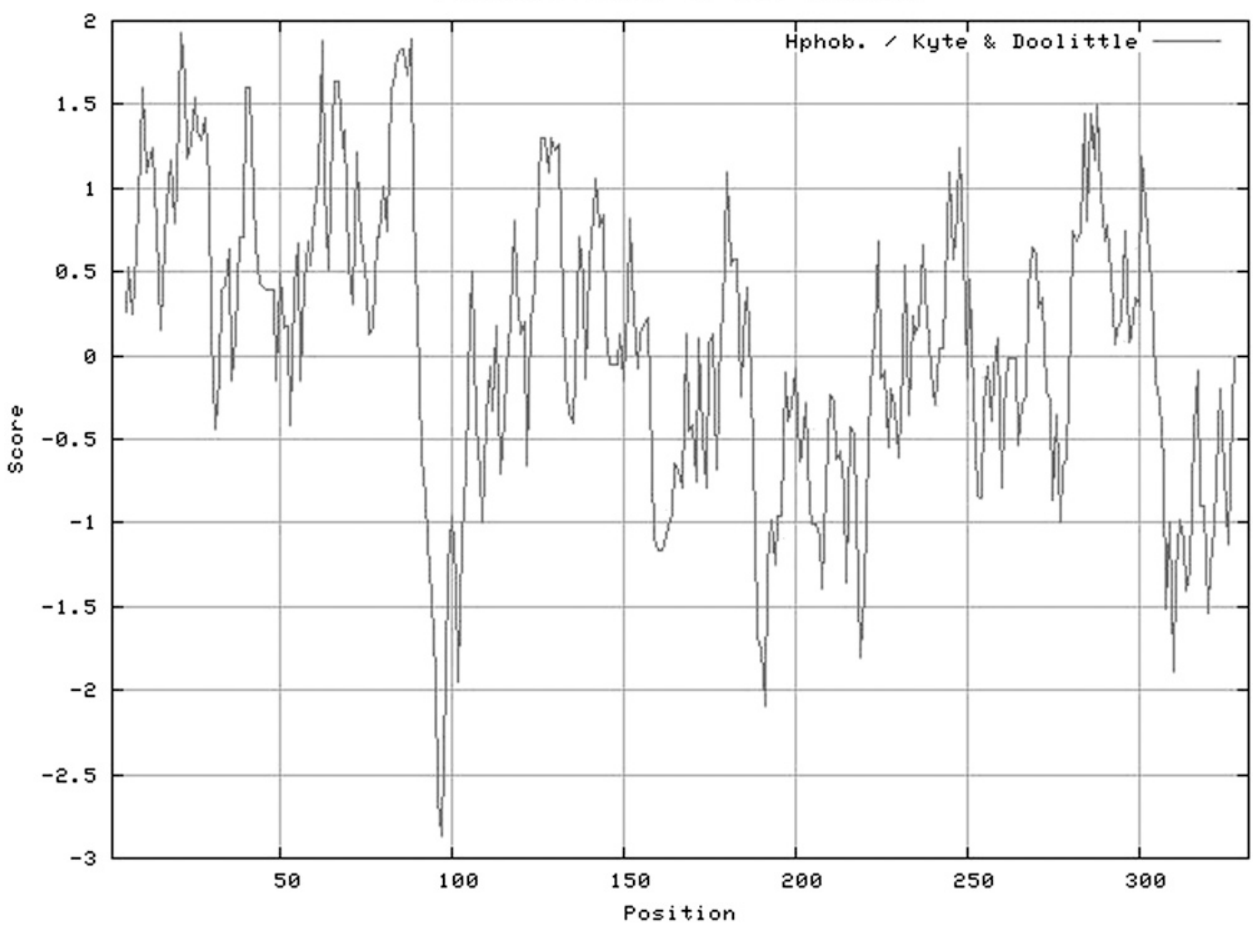

B
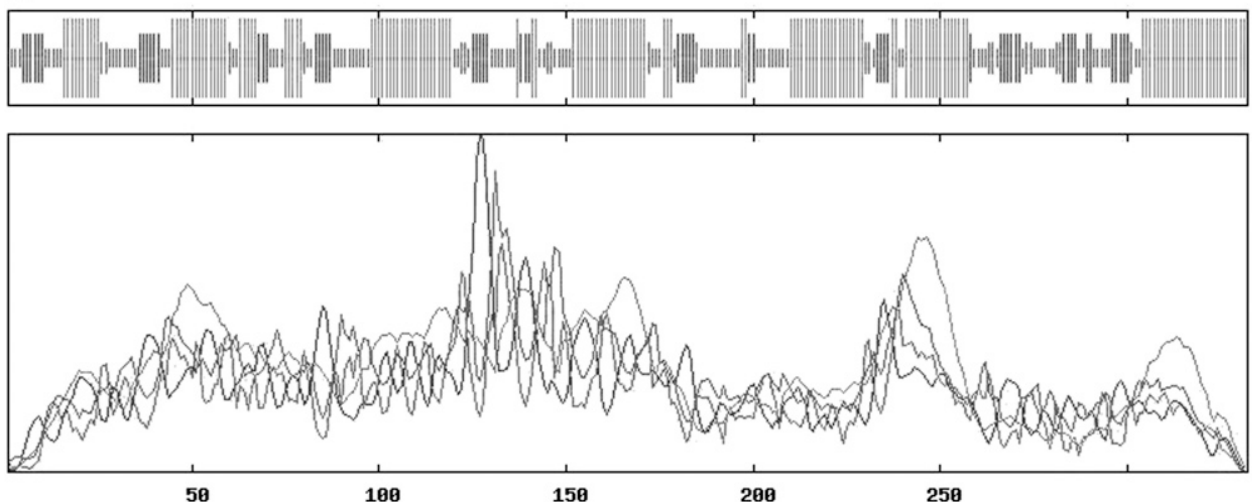

Fig. 3. (A) Hydropathy profile of MaMDH. Hydropathy was analyzed using ProtScale. Hydrophobic domains are those that are above the zero line, whereas the hydrophilic domains are those that are below the zero line. (B) MaMDH secondary structure prediction. The $\alpha$-helix was represented in blue. The $\beta$-turn was represented in green. The extended strand was represented in red. The random coil was represented in orange.

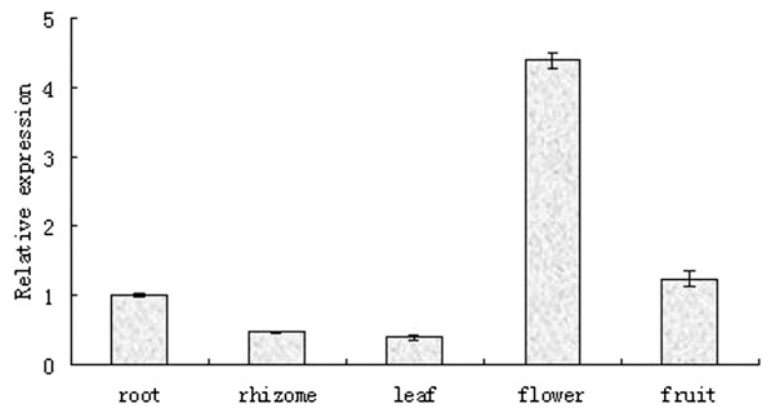

Fig. 4. Expression of $M a M D H$ in different organs by real-time reverse transcription-polymerase chain reaction. Samples were obtained from the roots, rhizomes, leaves, flowers, and fruits of 8-month-old plants. The means were generated from three independent measurements and the vertical bars indicate the sEs. When absent, the SE bars fall within the dimensions of the symbol.

In most climacteric fruits, ethylene evolution begins to increase at the onset of the climacteric period and continues to increase until the final decrease as the fully ripe stage is reached. As a typical climacteric fruit, banana fruits exhibit different ethylene biosynthesis patterns with a sharp rise and fall in ethylene production during the early climacteric rise in cell respiration (Liu et al., 1999). In this study there was no detectable ethylene production in naturally ripened banana fruits during the pre-climacteric period (Fig. 5A). A sharp peak in ethylene production at the onset of the climacteric period has been recognized as a characteristic ripening feature of banana fruits (Inaba et al., 2007). In ethylene-treated banana fruits, ethylene biosynthesis was activated immediately after ethylene treatment (Fig. 5B). 1-MCP inhibits ethylene perception (Sisler and Serek, 1997) and also influences ethylene biosynthesis by feedback inhibition of acc synthase gene (ACS) and acc oxidase gene (ACO) enzyme expression (Blankenship and Dole, 2003). Compared with ethylene-treated banana fruits, ethylene biosynthesis was almost completely inhibited in 1-MCP-treated banana fruits.

At present, there are many studies concerning MDH (Berkemeyer et al., 1998; Cvetić 

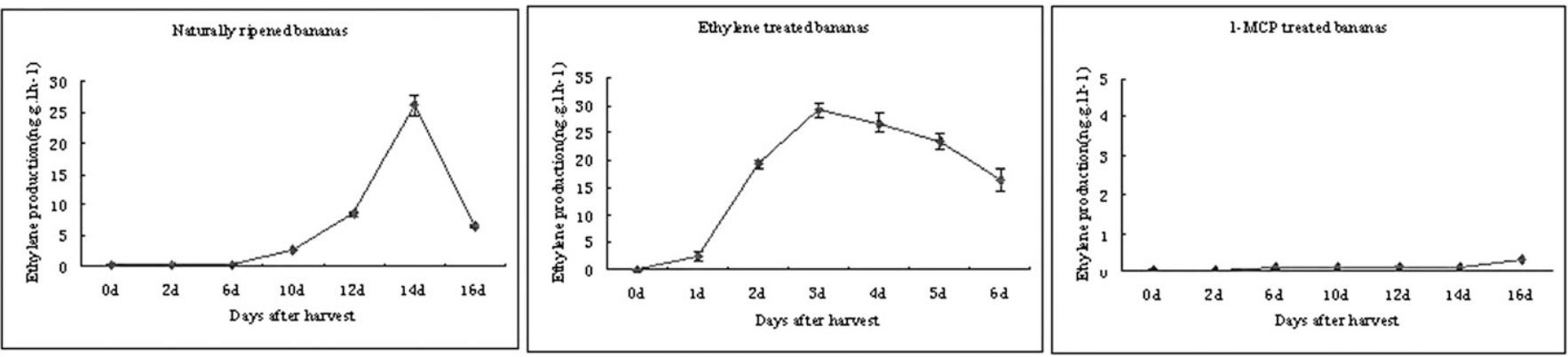

Fig. 5. Changes in ethylene production in naturally ripened (A), ethylene-treated (B), and 1-MCP-treated (C) bananas. Vertical bars indicate the sE of three replicates. When absent, the sE bars fall within the dimensions of the symbol. 1-MCP $=1$-methylcyclopropene.

A

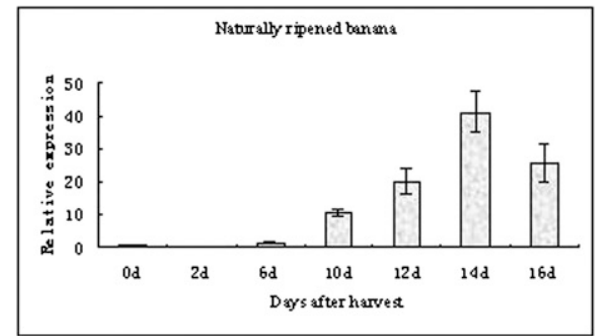

B

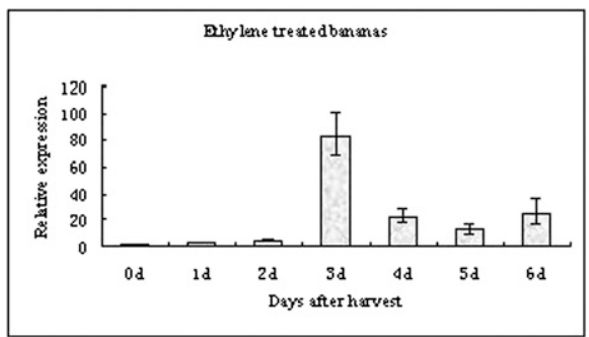

C

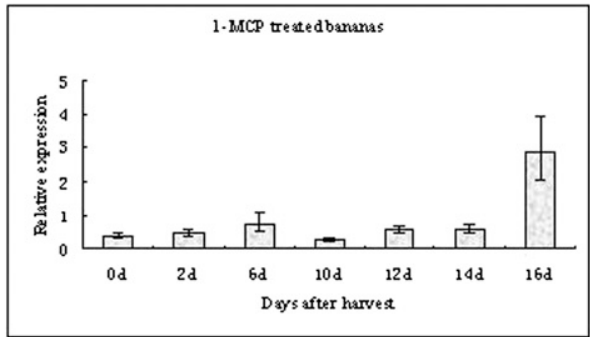

Fig. 6. Relative $M a M D H$ expression as determined by real-time reverse transcription-polymerase chain reaction in naturally ripened (A), ethylene-treated (B), and 1-MCP-treated $(\mathbf{C})$ bananas. The means were generated from three independent measurements and the vertical bars indicate the sEs. When absent, the sE bars fall within the dimensions of the symbol. 1-MCP $=1$-methylcyclopropene.

et al., 2008; Hebbelmann et al., 2012), but its function is not understood. One of the available studies about the various MDH types showed that $c y M D H$ expression in apples was higher in leaves, stems, and fruits accompanied by low expression in roots (Yao et al., 2011b). In this study, we followed $M a M D H$ expression in banana tissues using fluorescent quantitative PCR and found that the gene was expressed in roots, rhizomes, leaves, flowers, and fruits with the highest expression levels in the flowers followed by expression in roots and fruits and relatively low expression in rhizomes and leaves. Our results thus have similarities to that of Yao et al. (2011b), but some differences were also observed. Like apples, cytoplasmic malic acid dehydrogenase gene was expressed in all organs, but $M a M D H$ expression in the roots was considerably high; only flower- and fruitspecific expression preceded it. This difference may be a species characteristic in banana, but may also be the result of different mechanisms found in climacteric fruit.

To date, there are more studies on the decomposition and anabolic metabolism regulated by MDH, but the role of MDH in plant development and fruit ripening has not been fully clarified yet and our knowledge is still limited concerning cytoplasmic malate dehydrogenase gene expression during climacteric fruit ripening. We studied $M a M D H$ gene expression in naturally ripened banana fruits, 1-MCP-treated banana fruits, and ethylenetreated banana fruits (Fig. 6). In naturally ripened banana fruits, $M a M D H$ expression increased after harvest reaching a maximum on the same day as ethylene production peaked; in ethylene-treated banana fruits, $M a M D H$ expression rose sharply along with ethylene biosynthesis; in 1-MCP-treated banana fruits, $M a M D H$ expression was almost completely suppressed. This result suggests that $M a M D H$ expression may be developmentally regulated by ethylene biosynthesis during post-harvest banana fruit ripening. The relative maximal expression of $M a M D H$ during post-harvest ripening in ethylenetreated, naturally ripened, and 1-MCP-treated banana fruits was 83.1, 41.0 and 2.9, respectively. Exogenous ethylene treatment caused four-fold or greater and 28.6-fold or greater increases in maximum $\mathrm{MaMDH}$ expression compared with naturally ripened and 1-MCPtreated banana fruits, respectively (Fig. 6). These results strongly suggest that exogenous ethylene stimulated maximal $M a M D H$ expression. However, although endogenous ethylene biosynthesis is induced and peaked at Day 3, which is in line with $M a M D H$ expression, the expression of $M a M D H$ in ethylene-treated banana fruits changed sharply relative to the naturally ripened banana fruits. According to previous study (Liu et al., 1999), the change in ethylene production by the naturally ripened and ethylene-treated fruits was different and may be the result of different mechanisms involved in regulating ethylene biosynthesis. $M a M D H$ expression is likely induced by ethylene and plays an important role in the fruit ripening process, but additional data are needed to support this conclusion.

\section{Literature Cited}

Asif, M.H., P. Dhawan, and P. Nath. 2000. A simple procedure for the isolation of high quality RNA from ripening banana fruit. Plant Mol. Biol. Rpt. 18:109-115.

Berkemeyer, M., R. Scheibe, and O. Ocheretina. 1998. A novel, non-redox-regulated NADdependent malate dehydrogenase from chloroplasts of Arabidopsis thaliana L. J. Biol. Chem. 273:27927-27933.

Blankenship, S.M. and J.M. Dole. 2003. 1Methylcyclopropene: A review. Postharvest Biol. Technol. 28:1-25.

Cvetić, T., S. Veljović-Jovanović, and Z. Vucinić. 2008. Characterization of NAD-dependent malate dehydrogenases from spinach leaves. Protoplasma 232:247-253.

Ding, Y. and Q.H. Ma. 2004. Characterization of a cytosolicmalate dehydrogenase cDNA which encodes an isozyme tow and oxaloacetate reduction in wheat. Biochimie 86:509-518.

Etienne, C., A. Moing, E. Dirlewanger, P. Raymond, R. Monet, and C. Rothan. 2002. Isolation and characterization of six peach cDNAs encoding key proteins in organic acid metabolism and solute accumulation: Involvement in regulating peach fruit acidity. Physiol. Plant. 114:259-270.

Gietl, C. 1992. Partitioning of malate dehydrogenase isoenzymes into glyoxysomes, mitochondria, and chloroplasts. Plant Physiol. 100:557-559.

Giovannoni, J.J. 2004. Genetic Regulation of Fruit Development and Ripening. Plant Cell 16:170-180.

Gray, M.W., C. Burger, H.W. Leldt, and B.F. Lang. 2001. The origin and early evolution of mitochondria. Genome Biol. 2:10181-10185.

Hebbelmann, I., J. Selinski, C. Wehmeyer, T. Goss, I. Voss, P. Mulo, S. Kangasjärvi, E.M. Aro, M.L. Oelze, K.J. Dietz, A. Nunes-Nesi, P.T Do, A.R. Fernie, S.K. Talla, A.S. Raghavendra, 
V. Linke, and R. Scheibe. 2012. Multiple strategies to prevent oxidative stress in Arabidopsis plants lacking the malate valve enzyme NADP-malate dehydrogenase. J. Expt. Bot. 63:1445-1459.

Inaba, A., X. Liu, N. Yokotani, M. Yamane, W.J. Lu, R. Nakano, and Y. Kubo. 2007. Differential feedback regulation of ethylene biosynthesis in pulp and peel tissues of banana fruit. J. Expt. Bot. 58:1047-1057.

Kochianl, V., O.A. Hoekenga, and M.A. Pineros. 2004. How do crop plants tolerate acid soils? Mechanisms of aluminum tolerance and phosphorous efficiency. Annu. Rev. Plant Biol. 55:459-493.

Liu, X., S. Shiomi, A. Nakstsuka, Y. Kubo, R. Nakamura, and A. Inaba. 1999. Characterization of ethylene biosynthesis associated with ripening in banana fruit. Plant Physiol. 121: $1257-1265$.
Ma, J.F., P.R. Ryan, and E. Delhaize. 2001. Aluminium tolerance in plants and the complexing role of organic acids. Trends Plant Sci. 6:273-278.

McAlister-Henn, L. 1988. Evolutionary relationships among the malate dehydrogenases. Trends Biochem. Sci. 13:178-181.

Ocheretina, O. and R. Scheibe. 1997. Cloning and sequence analysis of cDNAs encoding plant cytosolic malate dehydrogenase. Gene 199:145-148.

Sisler, E.C. and M. Serek. 1997. Inhibitors of ethylene responses in plants at the receptor level: Recent developments. Physiol. Plant. 100:577-582.

Susan S.M., T.D. Brian, G.G. Robert, J.S.G., and P.V. Carroll. 1998. Alfalfa malate dehydrogenase (MDH): Molecular cloning and characterization of five different forms reveals a unique nodule-enhanced MDH. Plant J. 15:173-184.
Wang, Q.F., Y. Zhao, Q. Yi, K.Z. Li, Y.X. Yu, and L.M. Chen. 2010. Overexpression of malate dehydrogenase in transgenic tobacco leaves: Enhanced malate synthesis and augmented Alresistance. Acta Physiol. Plant. 32:1209-1220.

Xu, B.Y., W. Su, J.H. Liu, J.B. Wang, and Z.Q. Jin. 2007. Differentially expressed cDNAs at the early stage of banana ripening identified by suppression subtractive hybridization and cDNA microarray. Planta 226:529-539.

Yao, Y.X., Q.L. Dong, H. Zhai, C.X. You, and Y.J. Hao. 2011a. The functions of an apple cytosolic malate dehydrogenase gene in growth and tolerance to cold and salt stresses. Plant Physiol. Biochem. 49:257-264.

Yao, Y.X., M. Li, H. Zhai, C.X. You, and Y.J. Hao. 2011b. Isolation and characterization of an apple cytosolic malate dehydrogenase gene reveal its function in malate synthesis. J. Plant Physiol. 168:474-480. 